\title{
Elective Repeat Cesarean Compared with Spontaneous Trial of Labor after a Prior Cesarean: A Propensity Score Analysis
}

\author{
Sharon A. GILBERT, M.S., M.B.A. \\ The George Washington University Biostatistics Center, Washington, D.C. \\ William A. GROBMAN, M.D., M.B.A. \\ The Departments of Obstetrics and Gynecology at Northwestern University, Chicago, IL
}

\section{Mark B. LANDON, M.D.}

The Ohio State University, Columbus, $\mathrm{OH}$

Catherine Y. SPONG, M.D.

The Eunice Kennedy Shriver National Institute of Child Health and Human Development, Bethesda, MD

Dwight J. ROUSE, M.D.

University of Alabama at Birmingham, Birmingham, AL

Kenneth J. LEVENO, M.D.

University of Texas Southwestern Medical Center, Dallas, TX

Michael W. VARNER, M.D.

University of Utah, Salt Lake City, UT

Steve N. CARITIS, M.D.

University of Pittsburgh, Pittsburgh, PA

Paul J. MEIS, M.D.

Wake Forest University Health Sciences, Winston-Salem, NC

Yoram SOROKIN, M.D.

Wayne State University, Detroit, MI

Marshall CARPENTER, M.D.

Brown University, Providence, RI

Mary J. O'SULLIVAN, M.D.

University of Miami, Miami, FL

Baha M. SIBAI, M.D.

University of Tennessee, Memphis, TN

\footnotetext{
(C) 2012 Mosby, Inc. All rights reserved

Reprints will not be available Corresponding author: Sharon A. Gilbert, M.S., M.B.A. The George Washington University 6110 Executive Blvd, Suite 750 Rockville, MD 20852 301/881-9260 (phone) 301/816-0384 (fax) sgilbert@ bsc.gwu.edu.

*ther members of the Eunice Kennedy Shriver National Institute of Child Health and Human Development Maternal-Fetal Medicine Units Network are listed in the Acknowledgments.

Publisher's Disclaimer: This is a PDF file of an unedited manuscript that has been accepted for publication. As a service to our customers we are providing this early version of the manuscript. The manuscript will undergo copyediting, typesetting, and review of the resulting proof before it is published in its final citable form. Please note that during the production process errors may be discovered which could affect the content, and all legal disclaimers that apply to the journal pertain.

Disclosure: None of the authors have a conflict of interest.

Presented at the 32nd Annual Meeting of the Society for Maternal-Fetal Medicine. February 6-11, 2011. Dallas, TX.
} 
John M. THORP, M.D.

University of North Carolina at Chapel Hill, Chapel Hill, NC

Susan M. RAMIN, M.D.

The University of Texas Health Science Center at Houston, Houston, TX

Brian M. MERCER, M.D.

Case Western Reserve University-MetroHealth Medical Center, Cleveland, $\mathrm{OH}$

The Eunice Kennedy Shriver National Institute of Child Health and Human Development Maternal-Fetal Medicine Units Network

\begin{abstract}
Objective-To determine outcomes, after use of propensity score (PS) techniques to create balanced groups, according to whether a woman undergoes elective repeat cesarean (ERCD) or trial of labor (TOL).
\end{abstract}

Study Design-Women eligible for a TOL with 1 previous low transverse incision were categorized according to whether they underwent an ERCD or TOL. A PS technique was used to develop ERCD and TOL groups with comparable baseline characteristics. Outcomes were assessed using conditional logistic regression.

Results-Rates of endometritis, operative injury, respiratory distress syndrome and newborn infection were lower, while rates of hysterectomy and wound complication were higher, in the ERCD group.

Conclusion-PS techniques can be used to generate comparable ERCD and TOL groups. Some types of maternal morbidity, such as hysterectomy are higher, while others, such as operative injury, are lower in the ERCD group. Although the absolute risk is low, neonatal morbidity appears to be lower in the ERCD group.

\title{
Keywords
}

elective repeat cesarean; propensity score; trial of labor

\section{Introduction}

In March 2010 the Agency for Healthcare Research and Quality (AHRQ) published the results of an evidence report and technology assessment concerning women undergoing either a trial of labor (TOL) after previous cesarean or an elective repeat cesarean delivery (ERCD). ${ }^{1}$ The evidence report was based almost entirely on observational studies, and the lack of randomized trials to compare these two approaches to delivery was cited as a critical research gap. It also was noted that "several papers indicate that such a trial is unlikely in the United States", due both to the perception that pregnant women would not be willing to be randomized to a particular delivery approach and to the large sample size that would be required to power such a study adequately.

A randomized trial is considered the gold standard of clinical research given that, among other reasons, it is a design that optimizes the balance of observed as well as unobserved baseline covariates. Observational studies, conversely, are more prone to selection bias, often resulting in unbalanced baseline group differences. Various methods such as stratification, matching, and regression have been used to minimize this potential bias, however, these methods have limitations with regard to the number of covariates that can be incorporated. ${ }^{2}$ This limitation to control for confounding factors was specifically mentioned in the AHRQ Vaginal Birth After Cesarean evidence report. ${ }^{3}$ 
One statistical method introduced by Rosenbaum and Rubin, the propensity score, potentially circumvents these covariate restrictions. ${ }^{4}$ The propensity score is a single variable that represents the collection of baseline covariates for each individual or patient and is defined as the probability of being treated conditional on the individual's covariate values. This score, therefore, can be used to develop groups that differ according to the exposure of interest, but have otherwise similar baseline characteristics, and its use creates "the observational study analog of randomization in experiments". 5 Although several methods have been proposed to optimize inter-group similarity, Rosenbaum and Rubin found the nearest Mahalanobis metric matching within calipers defined by the propensity score to produce the best balance of baseline covariates between groups. ${ }^{6}$ Further discussion on propensity score methodology can be found in several papers and in textbooks. $7,8,9,10,11$

Given the past and projected absence of randomized trials comparing ERCD to TOL, propensity scores offer an alternate method to compare the outcomes related to the approach to delivery after a prior cesarean. This relatively new statistical technique has rarely been used in obstetrics and gynecology and does not appear to have been utilized in the context of TOL. ${ }^{12,13}$ Correspondingly, this analysis was designed to assess, using propensity score techniques, the maternal and neonatal outcomes associated with an ERCD compared with a TOL.

\section{Materials and Methods}

From 1999 through 2002, data were collected in a registry (the Cesarean Registry) by the Eunice Kennedy Shriver National Institute of Child Health and Human Development Maternal-Fetal Medicine Units Network. This observational study, which included data on all women with a prior cesarean who underwent delivery, was conducted at 19 clinical centers throughout the United States and was approved by the institutional review board of each participating center. Study personnel at the participating medical centers abstracted data from patient charts under a waiver of informed consent. Further detail on the Cesarean Registry can be obtained from previously published articles. ${ }^{14,15}$

This secondary analysis of the Cesarean Registry included women with a live, singleton, term, vertex gestation and one prior low transverse incision (LTCS) who had an ERCD or a TOL whose labor began spontaneously. Term was defined as greater than 36 weeks and 6 days gestation. An ERCD was defined as a cesarean delivery without any indication other than the prior cesarean. This group included women whose cesarean was scheduled, as well as those who chose cesarean, in the absence of any other indication, after presenting in labor or with rupture of membranes. Correspondingly, women who had a repeat cesarean for indications such as placenta previa or active herpes were excluded. Also, women were ineligible for the cohort if they had an ERCD prior to 39 weeks without spontaneous labor or premature rupture of membranes. Women with ERCD and spontaneous labor or rupture of membranes prior to 39 weeks were included since they were eligible for, and would need to choose between, the two approaches to delivery. Women with induced labor also were excluded since this intervention has been associated with a lower probability of success and a higher probability of uterine rupture, and is not purely a probabilistic possibility but a choice that a woman and her provider can make. ${ }^{16} \mathrm{~A}$ woman who is considering undergoing a spontaneous TOL or proceeding with an ERCD can only be counseled about the two approaches, either before or at the time of her labor, if the outcomes related to each alternative alone are known. This would be true, as well, if she were faced with the choice of undergoing a labor induction or an ERCD (in which case outcomes of induction versus ERCD would be studied). The suitability of analyzing women who are induced separately 
from those in spontaneous labor, in the appropriate circumstance, has been recognized in the literature. $17,18,19$

Propensity scores were used to control for potential covariate imbalances and create maximally non-biased groups. The propensity scores were generated using logistic regression and represent the probability of being delivered by an ERCD given baseline covariate values. Propensity scores were based on the ERCD group to ensure as many matches could be made as possible since the number of patients in this group was less than that in the TOL group. All covariates known at 37 weeks of gestation that existed prior to labor were included $(n=43)$ in the logistic regression without a stepwise procedure. This included 18 dichotomous variables that were used to represent the different centers from which patients were enrolled. One-to-one matching without replacement was conducted using the nearest Mahalanobis distance within calipers defined by the logit of the propensity score. The Mahalanobis distance was calculated by identifying, a priori, three variables that highly influenced the probability of cesarean delivery: maternal age, no prior vaginal delivery and recurrent cesarean indication (i.e., cephalopelvic disproportion (CPD) or failure to progress (FTP) as the indication for the prior cesarean). This matching method randomly ordered the treated subjects and chose the first subject in the list. All patients undergoing TOL who were within 0.25 standard deviation of the logit of the propensity score (or the caliper) of the identified subject in the ERCD group were selected. The pair with the smallest Mahalanobis distance was then removed and saved for use in analysis. The process was repeated until as many matches as possible could be made for the women in the ERCD group.

The two-sample Kolmogorov-Smirnov test was used to determine if the distributions of the propensity scores were similar between the two groups. ${ }^{20}$ The standardized difference was used to assess the balance of the covariates since it is a property of the sample, and unlike significance testing, does not depend upon the size of the sample. ${ }^{21}$ A standardized difference greater than the absolute value of 10 was used to indicate that the samples were meaningfully different. ${ }^{22}$ For the matched cohort, outcomes were compared using McNemar's test, and odds ratios (OR) and 95\% confidence intervals (CIs) were generated using conditional logistic regression. ${ }^{23}$ The CIs for number needed to treat were calculated using the Wilson score method. ${ }^{24}$

The evaluated maternal outcomes through delivery were: endometritis (clinical diagnosis of puerperal uterine infection in the absence of findings suggesting another source), wound complication (seroma, hematoma or infection), uterine rupture (disruption or tear of the uterine muscle and visceral peritoneum or a uterine muscle separation with extension to adjacent structures), operative injury (broad ligament hematoma, cystotomy, or bowel or ureteral injury), peripartum hysterectomy, thromboembolism (deep vein thrombosis or pulmonary embolus) and maternal death. Evaluated neonatal outcomes collected up to 120 days after delivery or at the time of hospital discharge were: transient tachypnea of the newborn (TTN), respiratory distress syndrome (RDS), infection including suspected or confirmed sepsis, acidemia (arterial cord pH less than 7.0), hypoxic ischemic encephalopathy (HIE), and neonatal death. Cases of uterine rupture, HIE and maternal death were confirmed by central review. For mothers and neonates, a separate composite adverse outcome variable representing one or more of these outcomes also was generated. An additional composite outcome that included only major maternal morbidities was developed and was defined as the occurrence of hysterectomy, uterine rupture or operative injury.

Statistical analysis was conducted with SAS (SAS Institute, Cary, NC) and, for the matched analysis, with the use of the R and the R MatchIt library (http://www.r-project.org/). A two- 
tailed nominal $\mathrm{p}<.05$ was considered significant. No adjustments were made for multiple comparisons. ${ }^{25}$

\section{Results}

Within the Cesarean Registry, 22,068 women with a live, singleton, vertex birth with one previous LTCS who delivered at term after undergoing a TOL or ERCD were available for analysis (Figure 1). Seven-hundred fifty-nine women who had indications for cesarean that would normally preclude a TOL, such as non-reassuring antepartum testing, malpresentation, placenta previa, myomectomy and active genital herpes were excluded. Similarly, in order to ensure that women who underwent repeat cesarean truly had no indication for the cesarean other than their choice, those who were reported to have a cesarean that was elective but who had a reported indication implying this was not the case (i.e. CPD, FTP, cord prolapse, non-reassuring tracing or abruption) were excluded $(n=262)$. An additional 3188 women who had an ERCD prior to 39 weeks of gestation as well as 3235 who underwent labor induction were not included in this analysis. Patients carrying fetuses with aneuploidy or congenital malformations, including trisomy 18 and 21, clubbed foot, cleft palate, and neural tube defect $(n=120)$ were removed as well. These women were excluded since the perinatal outcome of interest could be biased by their inclusion. This process left 14,504 women for analysis, of whom 8297 had a TOL and 6207 had an ERCD. Of the 8297 women who attempted a TOL, $6421(77.4 \%)$ had a successful vaginal birth after a previous cesarean (VBAC). Of the 6207 who had an ERCD, $4972(80.1 \%)$ delivered at or after 39 weeks of gestation without labor and 1235 (19.9\%) delivered between 37.0 and 38.6 weeks of gestation due to the onset of labor or premature rupture of membranes.

To generate the propensity scores for the probability of having an ERCD, the 25 baseline patient variables in Table 1 and 18 dichotomous center variables were used. First-level interactions of maternal age, no prior vaginal delivery and recurring indication for the prior cesarean were assessed with all of the variables. Twenty-two interactions were significant at the $\mathrm{p}<0.05$ level and were included in the final propensity score model. This resulted in 12,282 women ( $84.7 \%$ of the original cohort) available for further analysis since those with missing data for any variable were excluded from the logistic regression. The ERCD group consisted of 5500 women (44.8\%) and the TOL group 6782 (55.2\%).

First, we assessed whether the baseline characteristics of the groups, prior to matching, were similar. Figure 2 plots the distribution of the propensity scores in the two groups. Overall, as a function of baseline characteristics, the ERCD group had a higher probability of receiving an ERCD, as indicated by a higher mean propensity score $(0.576 \pm 0.189$ vs. $0.344 \pm 0.226$, $\mathrm{p}<.001)$. The initial difference in the two groups was further supported by the standardized difference criterion, which revealed that 17 out of the 43 (39.5\%) baseline covariates had a standardized difference of greater than 10 and were therefore imbalanced by this criterion. These differences, and the inherent selection bias they represent, supported the need for further ante hoc adjustment using propensity scores.

Even when two groups differ based on their baseline characteristics and corresponding mean propensity scores, the possibility that matched groups could be created is indicated when there is still overlap of the two groups' propensity scores. Figure 2 demonstrates that such a propensity score overlap between the two groups did exist. Thus, one-to-one Mahalanobis matching without replacement on the logit of the propensity score using calipers equal to 0.25 standard deviations of the propensity score was conducted to engender better covariate balance. This matching process resulted in the creation of 3981 matched pairs of ERCD and TOL patients. Figure 3 displays the distributions of the two matched groups' propensity scores, which in contrast to the distributions illustrated in Figure 2, reveal a high degree of 
overlap and similarity of shape between the two groups. This improved covariate balance also was reflected in the difference in the means of the propensity scores, which was reduced from 0.232 prior to propensity score-based matching to 0.022 post-matching (postmatching mean propensity scores of $0.512 \pm 0.176$ in the ERCD group and $0.490 \pm 0.168$ in the TOL group, $\mathrm{p}<.001$ ). The standardized difference criterion analysis further confirmed the groups' similarity as all baseline covariates were balanced and the highest standardized difference was only 7.5 (Table 2).

Table 3 presents the major maternal and neonatal outcomes, stratified by approach to delivery, of the matched cohort. The TOL success rate was $68.1 \%$ with 2710 women experiencing a successful VBAC. The ERCD group was less likely to have endometritis, operative injury, RDS, or newborn infection. Conversely, the ERCD group was more likely to have a wound complication or hysterectomy. Differences between the groups were not found for TTN, acidemia or neonatal death. All $31(0.8 \%)$ cases of uterine rupture occurred in the TOL group. One case each of thromboembolism and maternal death occurred in the ERCD group with none in the TOL group. The maternal death was attributed to amniotic fluid embolism. All three cases $(0.1 \%)$ of HIE occurred in the TOL group. Overall, the odds of maternal composite morbidity was $33 \%$ lower in the ERCD group (OR 0.67, 95\% CI $0.53-0.83$ ), while the odds of major maternal morbidity was $65 \%$ lower (OR $0.35,95 \%$ CI $0.20-0.62)$. The neonatal composite adverse outcome also was lower in the ERCD group by 33\% (OR 0.67, 95\% CI 0.55-0.80). This analysis implies that 62 women (95\% CI 40138) must undergo an ERCD to prevent one maternal adverse outcome, while 43 women (95\% CI 29-78) must undergo an ERCD to prevent one neonatal adverse outcome.

\section{Comment}

This analysis was undertaken to compare pregnancy outcomes in women with one prior LTCS undergoing ERCD or TOL after propensity scores were used to lessen potential selection bias and create two groups with similar observed baseline covariates. Indeed, this analysis demonstrated that prior to matching, the ERCD and TOL groups were significantly different with regard to baseline characteristics, but that after matching based on propensity scores, two groups of similar patients could be created. The highest standardized difference found after matching was 7.5, a value below the cut-off of 10 that indicates a meaningful inter-group difference. This analysis also demonstrated that even once confounding bias is minimized, some types of maternal morbidity, such as hysterectomy are higher, while others, such as operative injury, are lower in the ERCD group. Also, although the absolute risk is low, neonatal morbidity appears to be lower in the ERCD group. For the chosen composite outcomes, ERCD was associated with a lower odds of maternal and neonatal morbidity.

Several other studies have compared maternal and neonatal outcomes for women with a prior LTCS according to delivery approach. However, these studies may not have adequately controlled for patient characteristics. For example, in their investigation, McMahon et al. reported that an ERCD reduced major maternal complications, (rupture, hysterectomy or operative injury) by $44 \% .{ }^{26}$ However, the investigators controlled for only 7 baseline variables, leaving the potential for omitted variable bias. Moreover, simulation techniques by Peduzzi et al. have found that the validity of a logistic regression model may become problematic when the number of events relative to the number of independent variables is less than $10 .{ }^{27}$ The major composite outcome in the McMahon et al. analysis had 77 events, which was just enough to meet the Peduzzi et al. criterion; however, the analysis of three individual outcomes of hysterectomy $(\mathrm{n}=11)$, uterine rupture $(\mathrm{n}=11)$ and operative injury $(n=59)$ did not meet this criterion. 
Other studies have had even less adjustment for potential confounding factors. In an analysis of outcomes of 1755 women who underwent either a TOL or an ERCD, there was a significant difference between the groups for each of the eight baseline characteristics assessed. ${ }^{28}$ Yet, of the nine maternal outcomes reported, only 5 were evaluated in a multivariable analysis. Similarly, in a four-year prospective cohort study, in which maternal and infant outcomes were assessed according to delivery approach, only 3 baseline characteristics were compared and no adjustment was performed. ${ }^{29}$

Limitations of the present study should be noted. The results of this analysis may not apply to a population with different epidemiologic characteristics. Specifically, the matches were made by identifying women who underwent a TOL with spontaneous labor and who were similar to those who underwent an ERCD. This resulted in the final overall matched cohort being more similar, in terms of their baseline characteristics, to the original ERCD group. Most of the women in the matched cohort, for example, did not have a previous vaginal delivery, and this factor has been shown to lead to a higher TOL success rate as well as to a lower chance of maternal and neonatal morbidity. ${ }^{30}$ Thus, if there was a woman, or a group of women, who had a higher chance of achieving a vaginal birth after cesarean than the $68 \%$ of the group used in the matched analysis, the differences in outcomes could be less or even nonexistent. Also, even with a large observational study such as the Cesarean Registry there remains limited capacity to assess differences in rare maternal and neonatal outcomes (death, thromboembolism, hypoxic ischemic encephalopathy). A larger registry, such as that created on a national-level, may be able to better address these rare outcomes. Lastly, the composite outcomes, chosen based on prior morbidities that have been evaluated in the context of TOL studies, include morbidities with a range of clinical implications, and should be interpreted in conjunction with the individual outcome data.

It is important to note that the results of this analysis are only applicable for the comparison of the outcomes of spontaneous TOL and ERCD. This is a legitimate comparison to make, for although some women will require a labor induction, these women can choose at that point in time whether they wish to undergo a labor induction or ERCD. Although women cannot "choose" a spontaneous labor, they can choose to undertake TOL in that setting, and as such require data derived from the specific comparison of spontaneous TOL with ERCD. Conversely, counseling based on an aggregate population, which includes women who undergo labor induction, may give inaccurate insights into morbidity and outcomes for women with spontaneous labor. For example, induction has been shown to be associated with a higher risk of uterine rupture, postpartum hemorrhage, cesarean delivery and neonatal ICU admissions. ${ }^{31,32}$ That said, because labor induction has been associated with a lower rate of vaginal delivery and a higher rate of uterine rupture, the frequency of adverse outcome should only be greater among a general population of women undergoing an induced TOL, if such women were included or analyzed alone in comparison to ERCD.

Lastly, propensity score techniques can balance observed covariates but unlike a randomized trial, cannot control for unmeasured covariates. Nevertheless, because a randomized trial comparing ERCD to TOL of sufficient size likely will never be completed, techniques that ensure comparability between comparison groups are important to use in an effort to overcome inherent inter-group differences. The propensity score analysis presented here is such a technique that can be used to balance the groups on their observed covariates and generate a less biased comparison of outcomes.

\section{Acknowledgments}

The authors thank the following core committee members participated in protocol development and coordination between clinical research centers (Francee Johnson, BSN, Julia Gold BSN/APN), data management (Sandra Meadows), protocol/data management and statistical analysis (Elizabeth Thom, $\mathrm{PhD}$ and John C. Hauth, MD). 
In addition to the authors, other members of the Eunice Kennedy Shriver National Institute of Child Health and Human Development Maternal-Fetal Medicine Units Network are as follows:

The George Washington University Biostatistics Center - E. Thom, H. Juliussen-Stevenson, M. Fischer, L. Leuchtenburg

Northwestern University - A. Peaceman, M. Socol, D. Gradishar, G. Mallett

The Ohio State University — J. Iams, F. Johnson, S. Meadows, H. Walker

University of Alabama at Birmingham - J. Hauth, A. Northen, S. Tate

University of Texas Southwestern Medical Center - S. Bloom, J. Gold, D. Bradford

University of Utah - M. Belfort (Utah Valley Regional Medical Center), F. Porter (Intermountain Healthcare), B. Oshiro (McKay-Dee Hospital Center), K. Anderson (University of Utah Health Sciences Center), A. Guzman (McKay-Dee Hospital Center)

University of Pittsburgh - K. Lain, M. Cotroneo, D. Fischer, M. Luce

Wake Forest University Health Sciences - M. Harper, M. Swain, C. Moorefield, K. Lanier, L. Steele

Thomas Jefferson University - R. Wapner, A. Sciscione, M. DiVito, M. Talucci, M. Pollock

Wayne State University - M. Dombrowski, G. Norman, A. Millinder, C. Sudz, B. Steffy

University of Cincinnati - M. Miodovnik, T. Siddiqi, H. How, N. Elder

Columbia University — F. Malone, M. D'Alton, V. Pemberton, V. Carmona, H. Husami

Brown University - H. Silver, J. Tillinghast, D. Catlow, D. Allard

University of Miami - G. Burkett, J. Gilles, J. Potter, F. Doyle, S. Chandler

University of Tennessee - W. Mabie, R. Ramsey

University of Texas at San Antonio - O. Langer, D. Conway, S. Barker, M. Rodriguez

University of North Carolina - K. Moise, K. Dorman, S. Brody, J. Mitchell

The University of Texas Health Science Center at Houston - L. Gilstrap, M. Day, M. Kerr, E. Gildersleeve

Case Western Reserve University-MetroHealth Medical Center- P. Catalano, C. Milluzzi, B. Slivers, C. Santori

University of Chicago - A. Moawad, J. Hibbard, P. Jones, M. Ramos-Brinson, M. Moran, D. Scott

Eunice Kennedy Shriver National Institute of Child Health and Human Development - D. McNellis, K. Howell, S. Tolivaisa

MFMU Steering Committee Chair ( Vanderbilt University Medical Center) - S. Gabbe

The project described was supported by grants from the Eunice Kennedy Shriver National Institute of Child Health and Human Development (NICHD) of the National Institutes of Health (NIH) [HD21410, HD21414, HD27860, HD27861, HD27869, HD27905, HD27915, HD27917, HD34116, HD34122, HD34136, HD34208, HD34210, HD40500, HD40485, HD40544, HD40545, HD40560, HD40512, and HD36801] and its contents are solely the responsibility of the authors and do not necessarily represent the official view of NICHD or the NIH.

\section{References}

1. Guise, JM.; Eden, K.; Emeis, C., et al. AHRQ publication no. 10-E003. Agency for Healthcare Research and Quality; Rockville, MD: Mar. 2010 Vaginal birth after cesarean: new insights. Evidence/report technology assessment no. 191. (Prepared by the Oregon Health \& Science University Evidence-based Practice Center under contract no. 290-2007-10057-I.

2. Peduzzi P, Concato J, Kemper E, Holford TR, Feinstein AR. A simulation study of the number of events per variable in logistic regression analysis. J Clin Epidemiol. 1996; 49:1373-9. [PubMed: 8970487]

3. Guise, J-M.; McDonagh, M.; Hashima, J., et al. AHRQ publication no. 03-E018. Agency for Healthcare Research and Quality; Rockville, MD: Mar. 2003 Vaginal Birth After Cesarean (VBAC). Evidence report/technology Assessment no. 71 (Prepared by the Oregon Health \& Science University Evidence-based Practice Center under contract no 290-97-0018. 
4. Rosenbaum PR, Rubin DB. The central role of the propensity score in observational studies for causal effects. Biometrika. 1983; 70:41-55.

5. Rubin DB. On principles for modeling propensity scores in medical research. Pharmacoepidemiol Drug Safety. 2004; 13:855-7.

6. Rosenbaum PR, Rubin DB. Constructing a control group using multivariate matched sampling methods. Am Stat. 1985; 39:33-8.

7. Williamson E. Propensity scores: From naïve enthusiasm to intuitive understanding. Stat Methods Res. 2011 published online.

8. Braitman LE, Rosenbaum PR. Rare outcomes, common treatments: analytic strategies using propensity scores. Ann Intern Med. 2002; 137:693-5. [PubMed: 12379071]

9. D'Agostino RB. Tutorial in biostatistics propensity score methods for bias reduction in the comparison of a treatment to a non-randomized control group. Statist Med. 1998; 17:2265-81.

10. Guo, S.; Fraser, MW. Propensity Score Analysis, Statistical Methods and Applications. SAGE Publications; Thousand Oaks: 2010.

11. Rosenbaum, PR. Observational Studies. Springer-Verlag; New York: 2002.

12. Lewis BG, Halm EA, Marcus SM, Korenstein D, Federman AD. Preventive services use among women seen by gynecologists, general medical physicians, or both. Obstet Gynecol. 2008; 111(4): 945-52. [PubMed: 18378755]

13. Musselwhite KL, Faris P, Moore K, Berci D, King KM. Use of epideral anesthesia and the risk of acute postpartum urinary retention. Am J Obstet Gynecol. 2007; 196(5):472, e1-5. [PubMed: 17466708]

14. Landon MB, Hauth JC, Leveno KJ, et al. Maternal and perinatal outcomes associated with a trial of labor after prior cesarean delivery. N Engl J Med. 2004; 351:2581-9. [PubMed: 15598960]

15. Spong CY, Landon MB, Gilbert S, et al. Risk of uterine rupture and adverse perinatal outcome at term after cesarean delivery. Obstet Gynecol. 2007; 110:801-7. [PubMed: 17906012]

16. Induction of labor for vaginal birth after cesarean delivery. ACOG Committee Opinion No. 342. American College of Obstetricians and Gynecologists. Obstet Gynecol. 2006; 108:465-7. [PubMed: 16880321]

17. Grobman WA, Gilbert S, Landon MB, et al. Outcomes of induction of labor after one prior cesarean. Obstet Gynecol. 2007; 109:262-9. [PubMed: 17267822]

18. Delaney T, Young DC. Spontaneous versus induced labor after a previous cesarean delivery. Obstet Gynecol. 2003; 102:39-44. [PubMed: 12850605]

19. Lydon-Rochelle M, Holt VL, Easterling TR, Martin DP. Risk of uterine rupture during labor among women with a prior cesarean delivery. N Engl J Med. 2001; 345:3-8. [PubMed: 11439945]

20. Kiefer J. K-sample analogues of the Kolmogorov-Smirnov and Cramer-V. Mises tests. Annals of Mathematical Statistics. 1959; 30(2):420-447.

21. Austin PC. A critical appraisal of propensity-score matching in the medical literature between 1996 and 2003. Stat Med. 2008; 27:2037-49. [PubMed: 18038446]

22. Normand ST, Landrum MB, Guadagnoli E, et al. Validating recommendations for coronary angiography following acute myocardial infarction in the elderly: a matched analysis using propensity scores. J Clin Epidemiol. 2001; 54:387-98. [PubMed: 11297888]

23. McNemar Q. Note on the sampling error of the difference between correlated proportions or percentages. Psychometrika. 1947; 12(2):153-157. [PubMed: 20254758]

24. Bender R. Calculating confidence intervals for the number needed to treat. Control Clin Trials. 2001; 22:102-110. [PubMed: 11306148]

25. Rothman KJ. No adjustments are needed for multiple comparisons. Epidemiology. 1990; (1):43-6. [PubMed: 2081237]

26. McMahon MJ, Luther ER, Bowes WA, Olshan AF. Comparison of a trial of labor with an elective second cesarean section. N Engl J Med. 1996; 335:689-95. [PubMed: 8703167]

27. Peduzzi P, Concato J, Kemper E, Holford TR, Feinstein AR. A simulation study of the number of events per variable in logistic regression analysis. J Clin Epidemiol. 1996; 49:1373-9. [PubMed: 8970487] 
28. Hibbard JU, Ismail MA, Wang Y, Te C, Karrison T. Failed vaginal birth after a cesarean section: How risky is it? I. Maternal morbidity. Am J Obstet Gynecol. 2001; 184:1365-71. [PubMed: 11408854]

29. Blanchette H, Blanchette M, McCabe J, Vincent S. Is vaginal birth after cesarean safe? Experience at a community hospital. Am J Obstet Gynecol. 2001; 184:1478-84. [PubMed: 11408871]

30. Grobman WA, Lai YL, Landon MB, et al. Can a prediction model for vaginal birth after cesarean also predict the probability of morbidity related to a trial of labor? Am J Obstet Gynecol. 2009; 200:56, e1-e6. [PubMed: 18822401]

31. Delaney T, Young DC. Spontaneous versus induced labor after a previous cesarean delivery. Obstet Gynecol. 2003; 102:39-44. [PubMed: 12850605]

32. Lydon-Rochelle M, Holt VL, Easterling TR, Martin DP. Risk of uterine rupture during labor among women with a prior cesarean delivery. N Engl J Med. 2001; 345:3-8. [PubMed: 11439945] 


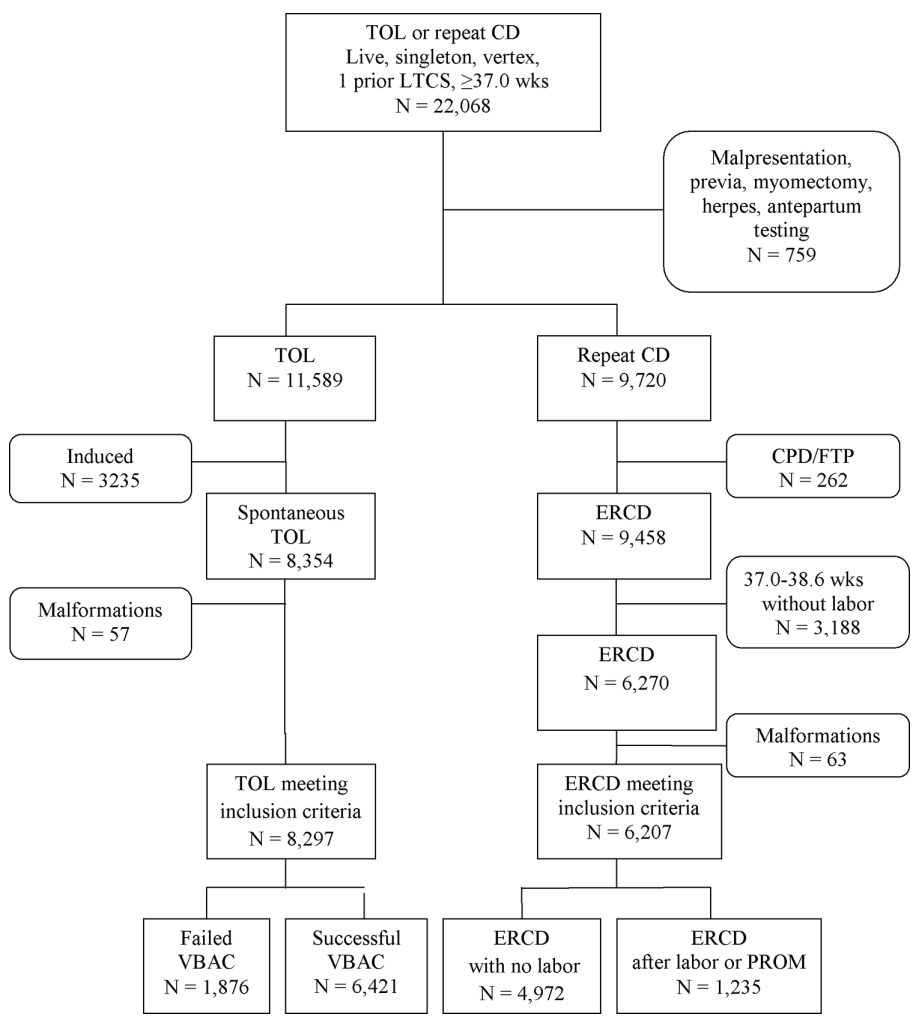

Figure 1.

Flow chart illustrating the development of the study groups.

LTCS, low transverse cesarean section; TOL, trial of labor; CD, cesarean delivery, CPD, cephalopelvic disproportion; FTP, failure to progress; ERCD, elective repeat cesarean delivery, VBAC, vaginal birth after cesarean 


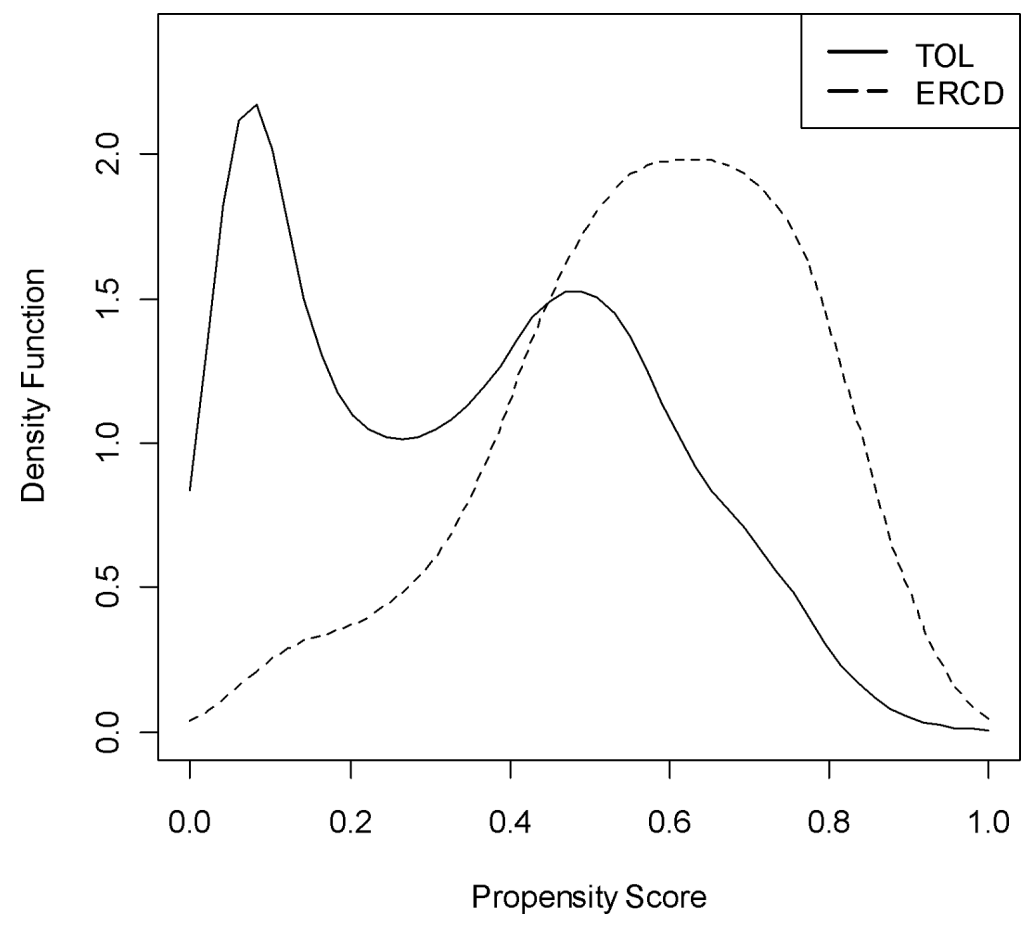

Figure 2.

Distribution of the propensity scores, prior to matching, stratified by approach to delivery. TOL, trial of labor; ERCD, elective repeat cesarean delivery 


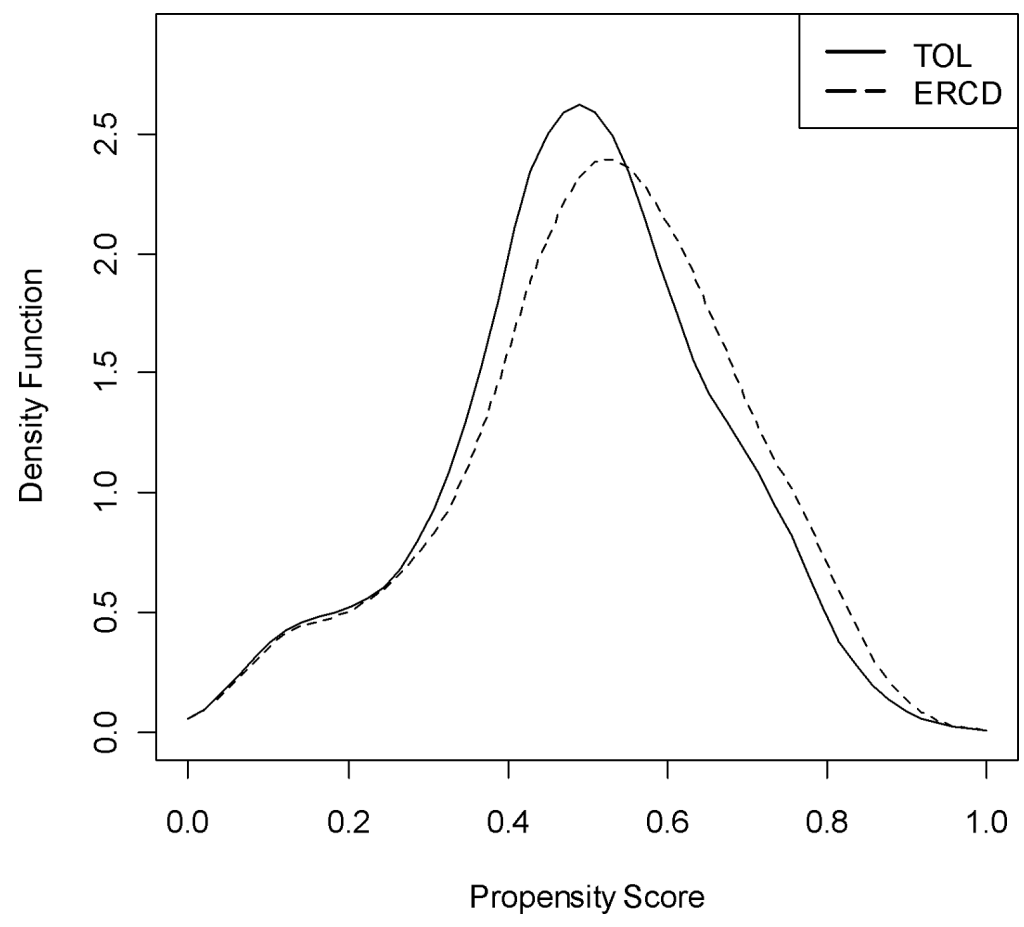

Figure 3.

Distribution of the propensity scores, after matching, stratified by approach to delivery. TOL, trial of labor; ERCD, elective repeat cesarean delivery 
Table 1

Baseline characteristics of the unmatched cohort, stratified by approach to delivery

\begin{tabular}{|c|c|c|c|}
\hline Characteristic & ERCD N = 5500 & TOL N = 6782 & SDif \\
\hline \multicolumn{4}{|l|}{ Demographics } \\
\hline Caucasian & $2703(49.1)$ & $2500(36.9)$ & 25.0 \\
\hline Married & $3597(65.4)$ & $3844(56.7)$ & 18.0 \\
\hline Prenatal care & $5466(99.4)$ & $6667(98.3)$ & 10.1 \\
\hline Private medical payment at delivery & $3018(54.9)$ & $2962(43.7)$ & 22.5 \\
\hline Body mass index at delivery & $32.4(28.7,37.4)$ & $30.6(27.2,34.8)$ & 31.2 \\
\hline Maternal age & $29(25,33)$ & $28(24,32)$ & 15.0 \\
\hline \multicolumn{4}{|l|}{ Past obstetric history } \\
\hline Prior cesarean indication of $\mathrm{CPD} / \mathrm{FTP} /$ failed forceps or vacuum & $3031(55.1)$ & $2474(36.5)$ & 38.1 \\
\hline$<2$ years since prior cesarean delivery & $577(10.5)$ & $566(8.3)$ & 7.3 \\
\hline No prior vaginal delivery & $4682(85.1)$ & $3681(54.3)$ & 71.3 \\
\hline No prior VBAC & $5235(95.2)$ & $4584(67.6)$ & 75.8 \\
\hline \multicolumn{4}{|l|}{ Current pregnancy history } \\
\hline EDC confirmation by first trimester US & $1814(33.0)$ & $1615(23.8)$ & 20.4 \\
\hline Diabetes & $437(7.9)$ & $240(3.5)$ & 19.0 \\
\hline Asthma & $380(6.9)$ & $493(7.3)$ & -1.4 \\
\hline Thyroid disease & $173(3.1)$ & $148(2.2)$ & 6.0 \\
\hline Seizure disorder & $38(0.7)$ & $43(0.6)$ & 0.7 \\
\hline Chronic hypertension & $70(1.3)$ & $40(0.6)$ & 7.1 \\
\hline Heart disease & $63(1.1)$ & $68(1.0)$ & 1.4 \\
\hline Cigarette use & $675(12.3)$ & $954(14.1)$ & -5.3 \\
\hline Alcohol use & $153(2.8)$ & $228(3.4)$ & -3.4 \\
\hline Street drug use & $108(2.0)$ & $263(3.9)$ & -11. \\
\hline Preterm labor requiring tocolysis & $60(1.1)$ & $124(1.8)$ & -6.2 \\
\hline Infection & $1502(27.3)$ & $2154(31.8)$ & -9.8 \\
\hline Antepartum antibiotic administration & $1233(22.4)$ & $1677(24.7)$ & -5.4 \\
\hline Gestational hypertension/preeclampsia & $208(3.8)$ & $178(2.6)$ & 6.6 \\
\hline Other maternal co-morbidity $^{a}$ & $85(1.5)$ & $160(2.4)$ & -5.9 \\
\hline
\end{tabular}

ERCD, elective repeat cesarean; TOL, trial of labor; SDiff, the standardized difference; CPD cephalopelvic disproportion; FTP, failure to progress; VBAC, vaginal birth after a prior cesarean; EDC, estimated date of confinement; US, ultrasound

Data presented as n $(\%)$ or median (interquartile range)

${ }^{a}$ Other maternal co-morbidity includes, prior to admission, abruption, pulmonary edema, any steroid use, renal disease or connective tissue disorder. 
Table 2

Baseline characteristics of the matched cohort, stratified by approach to delivery.

\begin{tabular}{|c|c|c|c|}
\hline Characteristic & ERCD N = 3981 & TOL N = 3981 & SDiff \\
\hline \multicolumn{4}{|l|}{ Demographics } \\
\hline Caucasian & $1700(42.7)$ & $1656(41.6)$ & 2.2 \\
\hline Married & $2485(62.4)$ & $2474(62.1)$ & 0.6 \\
\hline Prenatal care & $3951(99.2)$ & $3944(99.1)$ & 1.9 \\
\hline Private medical payment at delivery & 1979 (49.7) & $1939(48.7)$ & 2.0 \\
\hline Body mass index at delivery & $31.4(28.2,35.8)$ & $31.1(27.5,35.2)$ & 6.0 \\
\hline Maternal age & $29(24,33)$ & $28(24,33)$ & 2.0 \\
\hline \multicolumn{4}{|l|}{ Past obstetric history } \\
\hline Prior cesarean indication of $\mathrm{CPD} / \mathrm{FTP} /$ failed forceps or vacuum & $1887(47.4)$ & $1738(43.7)$ & 7.5 \\
\hline$<2$ years since last cesarean delivery & $436(11.0)$ & $422(10.6)$ & 1.1 \\
\hline No prior vaginal delivery & $3241(81.4)$ & 3209 (80.6) & 2.0 \\
\hline No prior VBAC & $3717(93.4)$ & $3711(93.2)$ & 0.6 \\
\hline \multicolumn{4}{|l|}{ Current pregnancy history } \\
\hline EDC confirmation by first trimester US & $1133(28.5)$ & $1094(27.5)$ & 2.2 \\
\hline Diabetes & $202(5.1)$ & $184(4.6)$ & 2.1 \\
\hline Asthma & $266(6.7)$ & $257(6.5)$ & 0.9 \\
\hline Thyroid disease & $102(2.6)$ & $101(2.5)$ & 0.2 \\
\hline Seizure disorder & $29(0.7)$ & $23(0.6)$ & 1.9 \\
\hline Chronic hypertension & $30(0.8)$ & $28(0.7)$ & 0.6 \\
\hline Heart disease & $41(1.0)$ & $42(1.1)$ & -0.2 \\
\hline Cigarette use & $448(11.3)$ & $433(10.9)$ & 1.2 \\
\hline Alcohol use & $110(2.8)$ & $107(2.7)$ & 0.5 \\
\hline Street drug use & $89(2.2)$ & $81(2.0)$ & 1.4 \\
\hline Preterm labor requiring tocolysis & $53(1.3)$ & $48(1.2)$ & 1.1 \\
\hline Infection & $1148(28.8)$ & $1177(29.6)$ & -1.6 \\
\hline Antepartum antibiotic administration & $920(23.1)$ & $935(23.5)$ & -0.9 \\
\hline Gestational hypertension/preeclampsia & $141(3.5)$ & $128(3.2)$ & 1.8 \\
\hline Other maternal co-morbidity ${ }^{a}$ & $69(1.7)$ & $72(1.8)$ & -0.6 \\
\hline
\end{tabular}

ERCD, elective repeat cesarean; TOL, trial of labor; SDiff, standardized difference; CPD, cephalopelvic disproportion; FTP, failure to progress; VBAC, vaginal birth after a prior cesarean; EDC, estimated date of confinement; US, ultrasound

Data represent $\mathrm{n}(\%)$ or median (1st, 3rd quartile)

ather maternal morbidity includes prior to admission, abruption, pulmonary edema, any steroid use, renal disease or connective tissue disorder. 
Table 3

Maternal and neonatal outcomes of the matched cohort, stratified by approach to delivery.

\begin{tabular}{|l|r|r|r|r|}
\hline Outcome & ERCD N =3981 N (\%) & TOL N = 3981 N (\%) & P-value $^{a}$ & Conditional OR (95\% CI) \\
\hline Maternal & & & & \\
Endometritis & $85(2.1)$ & $144(3.6)$ & $<.001$ & $0.57(0.43-0.75)$ \\
Wound complication & $34(0.9)$ & $17(0.4)$ & 0.02 & $2.00(1.12-3.58)$ \\
Operative injury & $6(0.2)$ & $20(0.5)$ & 0.006 & $0.30(0.12-0.75)$ \\
Hysterectomy & $12(0.3)$ & $3(0.1)$ & 0.02 & $4.00(1.13-14.17)$ \\
Maternal composite & $b$ & $200(5.0)$ & $<.001$ & $0.67(0.53-0.83)$ \\
Neonatal & $136(3.4)$ & & & \\
TTN & & & & \\
RDS & $119(3.0)$ & $107(2.7)$ & 0.41 & $1.12(0.86-1.46)$ \\
Infection & $41(1.0)$ & $68(1.7)$ & 0.01 & $0.60(0.41-0.89)$ \\
Acidemia & $124(3.1)$ & $211(5.3)$ & $<.001$ & $0.58(0.46-0.72)$ \\
Neonatal death & $14(0.4)$ & $25(0.6)$ & 0.08 & $0.56(0.29-1.08)$ \\
Neonatal composite & $c$ & $2(0.05)$ & 0.56 & $0.50(0.05-5.51)$ \\
\hline
\end{tabular}

ERCD, elective repeat cesarean; TOL, trial of labor; OR, odds ratio; CI, confidence interval; TTN, transient tachypnea of the newborn; RDS, respiratory distress

a P-value from McNemar's test

${ }^{b}$ Maternal composite outcome includes uterine rupture, endometritis, wound complication, operative injury, hysterectomy, thromboembolism or maternal death.

${ }^{c}$ Neonatal composite outcome includes TTN, RDS, infection, acidemia, neonatal death or hypoxic ischemic encephalopathy. 